\begin{tabular}{ccc}
\hline & International Journal of Engineering \& Technology, 9(2)(2020) 333-341 \\
SPC & Website: $w$ w w. sciencepubco.com/index.php/IJET \\
Research paper & Technology \\
\hline
\end{tabular}

\title{
Novel approach for anti-collision planning optimization in directional wells
}

\author{
Abdulrahman Mohamed * \\ Department of Petroleum Engineering and Gas Technology, Faculty of Energy and Environmental \\ Engineering, The British University in Egypt, Cairo, Egypt \\ *Corresponding author E-mail: abdulrahman.mohammed@hotmail.com
}

\begin{abstract}
One of the most application of the directional drilling is drilling multiple wells from one location or platform. In drilling multiple wells from one location the major problem that faced is avoiding the collision with the offset wells that drilled near the proposed well in the same region. Therefore, the Potential of Collison between the wells can cause severe catastrophic accidents such as an explosion or oil spill. Several measurements of proximity calculation or methods have been adopted to control the distance between the wells, avoid the Collison, increas-ing the clearance along with smoothing the trajectory, Reducing the drilling time based on the anti-collision rules. A real case study of an offshore directional horizontal well drilled from the platform is studied through the paper. The proposed well is drilled in the neighboring of three Offset wells that should be Planned completely to avoid the Collison with them. The well is planned through an advanced anti-collision method that results in preventing the collision of well with optimized drilling performance through Oriented separation factor (OSF). This factor yields appropriate separation with OSF greater than 5. This yield efficient separation with offset well 1 , offset well 2 and offset well 3 greater thant5, In addition to optimized drilling performance of $84 \%$ drilling versus $16 \%$ sliding that results in the completion of the well in 50 days with positive income that result in 8.55 Return on Investment (ROI).
\end{abstract}

Keywords: Anti-Collision Planning; Proximity Methods; Oriented Separation Factor; Anti-Collision Rules; Separation Factor; Alert Zones.

\section{Introduction}

Directional drilling is one of the most challenging and attractive techniques that help to solve many problems that vertical drilling cannot solve as drilling multiple wells from the same location in offshore drilling instead of having a platform for each well [1]. However, the drilling of multiple wells in the same location causes a high risk of Collision between the wells that can cause severe catastrophic accidents and loss of production. Therefore, the distance between the center of the offset and subject well must be calculated in order to avoid the collision by an offset well [2]. This can be achieved by accurate well planning taken into consideration separation from the offset wells. Another challenging problem is arising in determining the distance between the wells by the surveying because the surveying instrument does not give an accurate distance between the wells [3]. Poedjono [4] indicated that the center to center distance can be considered as the distance between the planned or subject well to the offset well which scanned by the minimum perpendicular method. Moreover, Spidle [5] stated that there is a more accurate method than the perpendicular method to estimate the accurate distance through horizontal distance measurement. Moreover, there is some uncertainty should be defined well and calculated to ensure the accurate positioning of the wellbore [6]. Therefore, gathering accurate information about the offset wells in the same working area is significant for avoiding the collision and for the future well planning [7]. In conclusion, another method than the calculation of the distance between the wells is to graph the subject well along with offset wells to control the collision in real-time [8].

\section{Methods}

The anti-collision planning methods were used for the field data of the subject well through determining the distance between the proposed well and offset wells. Then evaluating the distance between the wells through including the uncertainty of the surveying position through radii of uncertainty. After quantifying the separation between the wells, the well is classified and monitored through anti-collision rules to prevent the collision and redesign the high-risk segment of the wells. The more details of the used method are shown below.

\subsection{Proximity method used}

The first step in proximity is gathering information from the database of the working area to identify the offset wells that have high collision risk. These data showed three offset wells that show the high significant risk of collision. These wells were evaluated through the calculation of the distance between them and the proposed well through 3D least Distance Proximity method. This method is the most accurate method 
as it measures the shortest distance between the wells with high accuracy as it divided the well into small intervals and measure the shortest distance as shown in Fig.1 below shows the distance measurement method.

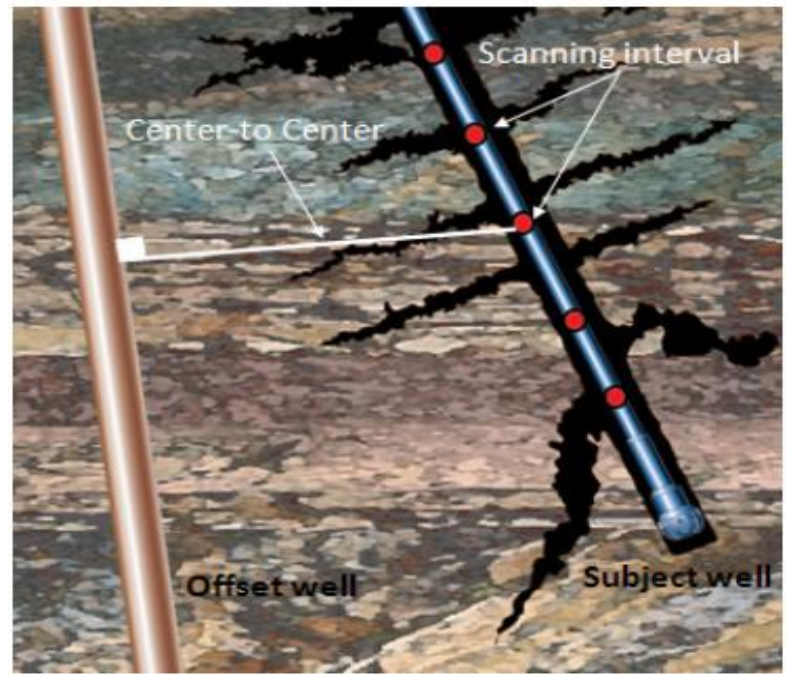

Fig. 1: 3D Least Distance Proximity Method.

\subsection{Proximity method used}

The proximity calculations were made after determining the distance between the offset well and subject well through proximity method. These method results in the center to center distance between wells but is not of high accuracy due to uncertainty of positioning of wells during the surveying method. Therefore, more proximity calculate is carried out to correct the separation between the wells as following.

\subsubsection{Separation factor $(\mathrm{SF})$}

$\mathrm{SF}$, Corrects the separation between the wells by considering the uncertainty of position between the subject well and the offset well. Mathematically, it represents the ratio between the center to center distance between the offset and subject well to the sum of the radii of the ellipsoid of uncertainty (EOU).

$\mathrm{SF}=\mathrm{S} /(\mathrm{er}+\mathrm{eo})$

Where S: the distance between the center to center of the reference and offset well, er: semi-major radius of the ellipsoid of the uncertainty of reference well and eo: semi-major radius of the ellipsoid of the uncertainty of offset well.

\subsubsection{Oriented separation factor (OSF)}

OSF provides a more accurate method for quantifying the separation between the wells and the EOR separation by taking into consideration the fixed probability of collision as described by SF equal to one. Furthermore, OSF considers the shape and geometry of the EOU resulting that all scenarios with the same SF have the same probability or chance of Collison. Mathematically, describes as in the following equation $(2)$;

OSF=Clearance/(Relative Positional Uncertainty)

\subsubsection{Allowable deviation from the plan (ADP)}

The allowable deviation from plane provides the drilling channel that created due to the avoidance of any proximity approach violation detected by oriented separation factor. It represented by distance radially from the plan at any point to the distance which the drilled can be allowed to deviate or depart from plan through drilling process in order to increase the drilling efficiency along without violating the anti-collision rules.

\subsubsection{Minimum allowable separation (MAS)}

The minimum allowable separation (MAS) measure the minimum distance between the center to center of the subject and offset well that is allowable with emphasizing on anti-collision rules. Therefore, the actual distance between the center to center between subject and offset wells during the normal drilling process can be obtained by summing the minimum allowable separation with allowable deviation from the plan.

\subsection{Anti-collision rules used based on proximity calculations}

Anti-collision planning between the offset wells and subject well is controlled through anti-collision rules that characterize and classify the risk degree of the collisions. These classifications are divided as alert zones, a minor risk well and major risk well as shown in the following Fig.2. 


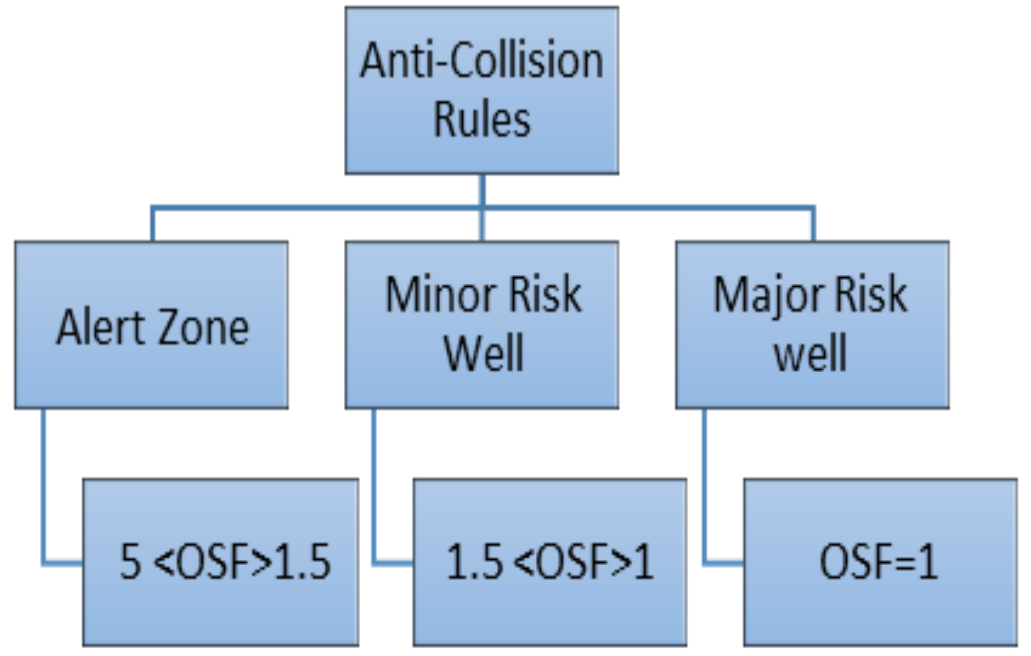

Fig. 2: Anti-Collision Rules.

\section{Results}

Real Case study of offshore horizontal well for Company X is used for anti-collision planning optimization through a novel method of OSF. The results below show the optimization and controlling of the directional well to control the collision through calculations and graphical methods.

\subsection{Results of the anti-collision proximity calculations of the offset wells 1}

Table 1: Clearance Calculations (Offset Well1)

\begin{tabular}{|c|c|c|c|c|c|c|c|c|c|c|}
\hline $\begin{array}{l}\text { Refer- } \\
\text { ence }\end{array}$ & $\begin{array}{l}\text { Refer- } \\
\text { ence }\end{array}$ & $\begin{array}{l}\text { Refer- } \\
\text { ence }\end{array}$ & Reference & Offset Well & Offset & Offset & Offset & Offset & $\begin{array}{l}\text { Angle } \\
\text { From }\end{array}$ & $\begin{array}{l}\text { Closest Ap- } \\
\text { proach Dis- } \\
\text { tance }\end{array}$ \\
\hline $\mathrm{MD}[\mathrm{ft}]$ & TVD $[\mathrm{ft}]$ & North $[\mathrm{ft}]$ & East[ft] & & $\mathrm{MD}[\mathrm{ft}]$ & $\begin{array}{l}\text { TVD } \\
{[\mathrm{ft}]}\end{array}$ & North[ft] & East[ft] & $\begin{array}{l}\text { High side } \\
\text { [deg] }\end{array}$ & {$[\mathrm{ft}]$} \\
\hline 82.02 & 82.02 & $0.00 \mathrm{~N}$ & $0.00 \mathrm{E}$ & $\begin{array}{l}\text { W \#1 - well Ab- } \\
\text { dulrahmanOFF1 }\end{array}$ & 82.02 & 82.02 & $0.00 \mathrm{~N}$ & $0.00 \mathrm{E}$ & 0 & 0 \\
\hline 278.87 & 278.87 & $0.00 \mathrm{~N}$ & $0.00 \mathrm{E}$ & $\begin{array}{l}\text { W \#1 - well Ab- } \\
\text { dulrahmanOFF1 }\end{array}$ & 278.86 & 278.86 & $0.95 \mathrm{~N}$ & $0.87 \mathrm{E}$ & 42.3 & 1.29 \\
\hline 393.7 & 393.7 & $0.00 \mathrm{~N}$ & $0.00 \mathrm{E}$ & $\begin{array}{l}\text { W \#1 - well Ab- } \\
\text { dulrahmanOFF1 }\end{array}$ & 393.65 & 393.63 & $2.42 \mathrm{~N}$ & $2.19 \mathrm{E}$ & 42.3 & 3.26 \\
\hline 1312.34 & 1310.05 & $56.05 \mathrm{~S}$ & $0.00 \mathrm{E}$ & $\begin{array}{l}\text { W \#1 - well Ab- } \\
\text { dulrahmanOFF1 }\end{array}$ & 1303.9 & 1302.55 & $36.70 \mathrm{~N}$ & $33.35 \mathrm{E}$ & -159.9 & 98.85 \\
\hline 1430.45 & 1427.28 & $70.44 \mathrm{~S}$ & $0.00 \mathrm{E}$ & $\begin{array}{l}\text { W \#1 - well Ab- } \\
\text { dulrahmanOFF1 }\end{array}$ & 1419.13 & 1417.36 & $43.95 \mathrm{~N}$ & $39.93 \mathrm{E}$ & -160.4 & 121.56 \\
\hline 2119.42 & 2114.55 & $112.47 \mathrm{~S}$ & $0.00 \mathrm{E}$ & $\begin{array}{l}\text { W \#1 - well Ab- } \\
\text { dulrahmanOFF1 }\end{array}$ & 2091.08 & 2085.12 & $99.05 \mathrm{~N}$ & $90.00 \mathrm{E}$ & 23.1 & 231.75 \\
\hline 2736.22 & 2731.34 & $112.47 \mathrm{~S}$ & $0.00 \mathrm{E}$ & $\begin{array}{l}\text { W \#1 - well Ab- } \\
\text { dulrahmanOFF1 }\end{array}$ & 2690.09 & 2677.07 & $166.71 \mathrm{~N}$ & $151.48 \mathrm{E}$ & 28.5 & 322.24 \\
\hline 4265.09 & 4260.22 & $112.47 \mathrm{~S}$ & $0.00 \mathrm{E}$ & $\begin{array}{l}\text { W \#1 - well Ab- } \\
\text { dulrahmanOFF1 }\end{array}$ & 4138.37 & 4089.54 & $401.72 \mathrm{~N}$ & $365.03 \mathrm{E}$ & 35.4 & 653.28 \\
\hline 4429.13 & 4424.26 & $112.47 \mathrm{~S}$ & $0.00 \mathrm{E}$ & $\begin{array}{l}\text { W \#1 - well Ab- } \\
\text { dulrahmanOFF1 }\end{array}$ & 4287.53 & 4233.1 & $431.67 \mathrm{~N}$ & $392.24 \mathrm{E}$ & 35.8 & 697.48 \\
\hline 4986.88 & 4975.88 & $72.39 \mathrm{~S}$ & $58.96 \mathrm{~W}$ & $\begin{array}{l}\text { W \#1 - well Ab- } \\
\text { dulrahmanOFF1 }\end{array}$ & 4786.73 & 4710.61 & $539.33 \mathrm{~N}$ & $490.07 \mathrm{E}$ & 92.7 & 863.71 \\
\hline 7208.01 & 6502.9 & $758.72 \mathrm{~N}$ & $1281.44 \mathrm{~W}$ & $\begin{array}{l}\text { W \#1 - well Ab- } \\
\text { dulrahmanOFF1 }\end{array}$ & 6042.34 & 5888.05 & $861.31 \mathrm{~N}$ & $782.63 \mathrm{E}$ & 84.6 & 2156.15 \\
\hline 7381.89 & 6545.82 & $853.43 \mathrm{~N}$ & $1420.75 \mathrm{~W}$ & $\begin{array}{l}\text { W \#1 - well Ab- } \\
\text { dulrahmanOFF1 }\end{array}$ & 6072.78 & 5916.12 & $870.03 \mathrm{~N}$ & $790.56 \mathrm{E}$ & 79.3 & 2299.28 \\
\hline 7665.62 & 6586.53 & $1011.18 \mathrm{~N}$ & $1652.79 \mathrm{~W}$ & $\begin{array}{l}\text { W \#1 - well Ab- } \\
\text { dulrahmanOFF1 }\end{array}$ & 6095.1 & 5936.7 & $876.43 \mathrm{~N}$ & $796.37 \mathrm{E}$ & 69.1 & 2537.49 \\
\hline 7988.68 & 6611.93 & $1192.26 \mathrm{~N}$ & 1919.13W & $\begin{array}{l}\text { W \#1 - well Ab- } \\
\text { dulrahmanOFF1 }\end{array}$ & 6101.06 & 5942.19 & $878.14 \mathrm{~N}$ & $797.92 \mathrm{E}$ & 69.4 & 2815.96 \\
\hline 8087.66 & 6617.48 & $1247.58 \mathrm{~N}$ & $2001.02 \mathrm{~W}$ & $\begin{array}{l}\text { W \#1 - well Ab- } \\
\text { dulrahmanOFF1 }\end{array}$ & 6100.7 & 5941.86 & $878.03 \mathrm{~N}$ & $797.83 \mathrm{E}$ & 64.8 & 2902.85 \\
\hline 9071.29 & 6650.26 & $1795.60 \mathrm{~N}$ & $2817.18 \mathrm{~W}$ & $\begin{array}{l}\text { W \#1 - well Ab- } \\
\text { dulrahmanOFF1 }\end{array}$ & 6075.45 & 5918.58 & $870.80 \mathrm{~N}$ & $791.26 \mathrm{E}$ & 63.7 & 3796.24 \\
\hline
\end{tabular}

Table 1 shows the clearance calculations between the subject well and the offset well 1 with respect of MD, TVD, North and East coordinates of both wells. In addition to the closest distance between the two wells. From the calculations, it shows that distance between the subject and offset well 1 were $1.29 \mathrm{ft}$ at $278.87 \mathrm{ft} \mathrm{MD}$ and increase gradually till reaching the target at $3796.24 \mathrm{ft}$ at $9071.29 \mathrm{MD}$. This indicated that the separation between the two wells was to low and increase as deepening of the well according to the OSF method that must keep OSF greater than 5 . 
3.2. Results of the anti-collision proximity calculations of the offset wells 2

Table 2: Clearance Calculations (Offset Well 2)

\begin{tabular}{|c|c|c|c|c|c|c|c|c|c|c|}
\hline $\begin{array}{l}\text { Refer- } \\
\text { ence }\end{array}$ & $\begin{array}{l}\text { Refer- } \\
\text { ence }\end{array}$ & $\begin{array}{l}\text { Refer- } \\
\text { ence }\end{array}$ & Reference & & Offset & Offset & Offset & Offset & $\begin{array}{l}\text { Angle } \\
\text { From }\end{array}$ & $\begin{array}{l}\text { Closest Ap- } \\
\text { proach Dis- } \\
\text { tance }\end{array}$ \\
\hline $\mathrm{MD}[\mathrm{ft}]$ & $\begin{array}{l}\text { TVD } \\
{[\mathrm{ft}]}\end{array}$ & North[ft] & East[ft] & Ottset Well & $\mathrm{MD}$ [ft] & $\begin{array}{l}\text { TVD } \\
{[\mathrm{ft}]}\end{array}$ & North[ft] & East[ft] & $\begin{array}{l}\text { High } \\
\text { side } \\
{[\mathrm{deg}]}\end{array}$ & {$[\mathrm{ft}]$} \\
\hline 82.02 & 82.02 & $0.00 \mathrm{~N}$ & $0.00 \mathrm{E}$ & $\begin{array}{l}\text { W \#1 - well Ab- } \\
\text { dulrahmanOFF2 }\end{array}$ & 82.02 & 82.02 & $0.00 \mathrm{~N}$ & $0.00 \mathrm{E}$ & 0 & 0 \\
\hline 278.87 & 278.87 & $0.00 \mathrm{~N}$ & $0.00 \mathrm{E}$ & $\begin{array}{l}\text { W \#1 - well Ab- } \\
\text { dulrahmanOFF2 }\end{array}$ & 278.77 & 278.71 & $3.79 \mathrm{~N}$ & $1.97 \mathrm{E}$ & 27.4 & 4.27 \\
\hline 393.7 & 393.7 & $0.00 \mathrm{~N}$ & $0.00 \mathrm{E}$ & $\begin{array}{l}\text { W \#1 - well Ab- } \\
\text { dulrahmanOFF2 }\end{array}$ & 393.19 & 392.94 & $9.51 \mathrm{~N}$ & $4.93 \mathrm{E}$ & 27.4 & 10.74 \\
\hline 1312.34 & 1310.05 & $56.05 \mathrm{~S}$ & $0.00 \mathrm{E}$ & $\begin{array}{l}\text { W \#1 - well Ab- } \\
\text { dulrahmanOFF2 }\end{array}$ & 1269.51 & 1251.88 & $76.44 \mathrm{~N}$ & $146.50 \mathrm{E}$ & -130.3 & 205.92 \\
\hline 1430.45 & 1427.28 & $70.44 \mathrm{~S}$ & $0.00 \mathrm{E}$ & $\begin{array}{l}\text { W \#1 - well Ab- } \\
\text { dulrahmanOFF2 }\end{array}$ & 1373.94 & 1350.33 & $84.35 \mathrm{~N}$ & $180.37 \mathrm{E}$ & -128.7 & 249.83 \\
\hline 2119.42 & 2114.55 & $112.47 \mathrm{~S}$ & $0.00 \mathrm{E}$ & $\begin{array}{l}\text { W \#1 - well Ab- } \\
\text { dulrahmanOFF2 }\end{array}$ & 1926.83 & 1847.11 & $124.75 \mathrm{~N}$ & $417.71 \mathrm{E}$ & 60.4 & 549.8 \\
\hline 2736.22 & 2731.34 & $112.47 \mathrm{~S}$ & $0.00 \mathrm{E}$ & $\begin{array}{l}\text { W \#1 - well Ab- } \\
\text { dulrahmanOFF2 }\end{array}$ & 2373.97 & 2214.07 & $154.73 \mathrm{~N}$ & $671.09 \mathrm{E}$ & 68.3 & 888.45 \\
\hline 4265.09 & 4260.22 & $112.47 \mathrm{~S}$ & $0.00 \mathrm{E}$ & $\begin{array}{l}\text { W \#1 - well Ab- } \\
\text { dulrahmanOFF2 }\end{array}$ & 3491.75 & 3076.43 & $207.91 \mathrm{~N}$ & $1379.74 \mathrm{E}$ & 76.9 & 1845.98 \\
\hline 4429.13 & 4424.26 & $112.47 \mathrm{~S}$ & $0.00 \mathrm{E}$ & $\begin{array}{l}\text { W \#1 - well Ab- } \\
\text { dulrahmanOFF2 }\end{array}$ & 3628.43 & 3180.79 & $210.90 \mathrm{~N}$ & $1467.95 \mathrm{E}$ & 77.6 & 1950.81 \\
\hline 4986.88 & 4975.88 & $72.39 \mathrm{~S}$ & $58.96 \mathrm{~W}$ & $\begin{array}{l}\text { W \#1 - well Ab- } \\
\text { dulrahmanOFF2 }\end{array}$ & 3988.14 & 3455.64 & $214.97 \mathrm{~N}$ & $1699.96 \mathrm{E}$ & 125.2 & 2342.55 \\
\hline 7208.01 & 6502.9 & $758.72 \mathrm{~N}$ & $1281.44 \mathrm{~W}$ & $\begin{array}{l}\text { W \#1 - well Ab- } \\
\text { dulrahmanOFF2 }\end{array}$ & 4331.99 & 3714.32 & $216.86 \mathrm{~N}$ & $1926.49 \mathrm{E}$ & 36.6 & 4284.93 \\
\hline 7381.89 & 6545.82 & $853.43 \mathrm{~N}$ & $1420.75 \mathrm{~W}$ & $\begin{array}{l}\text { W \#1 - well Ab- } \\
\text { dulrahmanOFF2 }\end{array}$ & 4252.58 & 3654.5 & $216.45 \mathrm{~N}$ & $1874.26 \mathrm{E}$ & 31.2 & 4429.74 \\
\hline 7665.62 & 6586.53 & $1011.18 \mathrm{~N}$ & $1652.79 \mathrm{~W}$ & $\begin{array}{l}\text { W \#1 - well Ab- } \\
\text { dulrahmanOFF2 }\end{array}$ & 4120.73 & 3555.73 & $215.81 \mathrm{~N}$ & $1786.93 \mathrm{E}$ & 24.8 & 4652.96 \\
\hline 7988.68 & 6611.93 & $1192.26 \mathrm{~N}$ & 1919.13W & $\begin{array}{l}\text { W \#1 - well Ab- } \\
\text { dulrahmanOFF2 }\end{array}$ & 4020.33 & 3480.01 & $215.19 \mathrm{~N}$ & $1720.99 \mathrm{E}$ & 23.6 & 4900.42 \\
\hline 8087.66 & 6617.48 & $1247.58 \mathrm{~N}$ & $2001.02 \mathrm{~W}$ & $\begin{array}{l}\text { W \#1 - well Ab- } \\
\text { dulrahmanOFF2 }\end{array}$ & 3971.42 & 3442.98 & $214.86 \mathrm{~N}$ & $1689.04 \mathrm{E}$ & 21.5 & 4975.99 \\
\hline 9071.29 & 6650.26 & $1795.60 \mathrm{~N}$ & 2817.18W & $\begin{array}{l}\text { W \#1 - well Ab- } \\
\text { dulrahmanOFF2 }\end{array}$ & 3545.91 & 3117.77 & $209.16 \mathrm{~N}$ & $1414.71 \mathrm{E}$ & 17.1 & 5736.22 \\
\hline
\end{tabular}

Table 2 Also, shows the clearance calculations between the subject well and the offset well 2 with respect of MD, TVD, North and East coordinates of both wells. In addition to the closest distance between the two wells. Accordingly, it shows that distance between the subject and offset well 2 were $4.27 \mathrm{ft}$ at $278.87 \mathrm{ft}$ MD and increase gradually till reaching the target at $5736.22 \mathrm{ft}$ at $9071.29 \mathrm{MD}$. This indicated that the separation between the two wells was to low and increase as deepening of the well according to the OSF method that must keep OSF greater than 5 .

\subsection{Results of the anti-collision proximity calculations of the offset wells 3}

Table 3: Clearance Report (Offset Well3)

\begin{tabular}{|c|c|c|c|c|c|c|c|c|c|c|}
\hline $\begin{array}{l}\text { Refer- } \\
\text { ence }\end{array}$ & $\begin{array}{l}\text { Refer- } \\
\text { ence }\end{array}$ & $\begin{array}{l}\text { Refer- } \\
\text { ence }\end{array}$ & Reference & Offset Well & Offset & Offset & Offset & Offset & $\begin{array}{l}\text { Angle } \\
\text { From }\end{array}$ & $\begin{array}{l}\text { Closest Ap- } \\
\text { proach Dis- } \\
\text { tance }\end{array}$ \\
\hline $\mathrm{MD}[\mathrm{ft}]$ & TVD [ft] & North[ft] & East $[\mathrm{ft}]$ & Omset well & $\mathrm{MD}[\mathrm{ft}]$ & $\begin{array}{l}\text { TVD } \\
{[\mathrm{ft}]}\end{array}$ & North[ft] & East[ft] & $\begin{array}{l}\text { High } \\
\text { side } \\
{[\mathrm{deg}]}\end{array}$ & {$[\mathrm{ft}]$} \\
\hline 82.02 & 82.02 & $0.00 \mathrm{~N}$ & $0.00 \mathrm{E}$ & $\begin{array}{l}\text { W \#1 - well Ab- } \\
\text { dulrahmanOFF3 }\end{array}$ & 82.02 & 82.02 & $0.00 \mathrm{~N}$ & $0.00 \mathrm{E}$ & 0 & 0 \\
\hline 278.87 & 278.87 & $0.00 \mathrm{~N}$ & $0.00 \mathrm{E}$ & $\begin{array}{l}\text { W \#1 - well Ab- } \\
\text { dulrahmanOFF3 }\end{array}$ & 278.83 & 278.81 & $1.35 \mathrm{~N}$ & $2.26 \mathrm{E}$ & 59.2 & 2.63 \\
\hline 393.7 & 393.7 & $0.00 \mathrm{~N}$ & $0.00 \mathrm{E}$ & $\begin{array}{l}\text { W \#1 - well Ab- } \\
\text { dulrahmanOFF3 }\end{array}$ & 393.52 & 393.43 & $3.38 \mathrm{~N}$ & $5.67 \mathrm{E}$ & 59.2 & 6.61 \\
\hline 1312.34 & 1310.05 & $56.05 \mathrm{~S}$ & $0.00 \mathrm{E}$ & $\begin{array}{l}\text { W \#1 - well Ab- } \\
\text { dulrahmanOFF3 }\end{array}$ & 1287.93 & 1279.93 & $50.46 \mathrm{~N}$ & $101.55 \mathrm{E}$ & -135.1 & 150.21 \\
\hline 1430.45 & 1427.28 & $70.44 \mathrm{~S}$ & $0.00 \mathrm{E}$ & $\begin{array}{l}\text { W \#1 - well Ab- } \\
\text { dulrahmanOFF3 }\end{array}$ & 1396.67 & 1385.83 & $60.69 \mathrm{~N}$ & $123.96 \mathrm{E}$ & -135.3 & 185.15 \\
\hline 2119.42 & 2114.55 & $112.47 \mathrm{~S}$ & $0.00 \mathrm{E}$ & $\begin{array}{l}\text { W \#1 - well Ab- } \\
\text { dulrahmanOFF3 }\end{array}$ & 2034.88 & 2003.05 & $150.11 \mathrm{~N}$ & $257.67 \mathrm{E}$ & 44.5 & 384.41 \\
\hline 2736.22 & 2731.34 & $112.47 \mathrm{~S}$ & $0.00 \mathrm{E}$ & $\begin{array}{l}\text { W \#1 - well Ab- } \\
\text { dulrahmanOFF3 }\end{array}$ & 2627.91 & 2567.68 & $300.38 \mathrm{~N}$ & $356.03 \mathrm{E}$ & 40.8 & 569.2 \\
\hline 4265.09 & 4260.22 & $112.47 \mathrm{~S}$ & $0.00 \mathrm{E}$ & $\begin{array}{l}\text { W \#1 - well Ab- } \\
\text { dulrahmanOFF3 }\end{array}$ & 4205.45 & 4062.25 & $742.51 \mathrm{~N}$ & $186.78 \mathrm{E}$ & 12.3 & 897.26 \\
\hline 4429.13 & 4424.26 & $112.47 \mathrm{~S}$ & $0.00 \mathrm{E}$ & $\begin{array}{l}\text { W \#1 - well Ab- } \\
\text { dulrahmanOFF3 }\end{array}$ & 4370.83 & 4216.88 & $786.20 \mathrm{~N}$ & $147.66 \mathrm{E}$ & 9.3 & 934.04 \\
\hline 4986.88 & 4975.88 & $72.39 \mathrm{~S}$ & $58.96 \mathrm{~W}$ & $\begin{array}{l}\text { W \#1 - well Ab- } \\
\text { dulrahmanOFF3 }\end{array}$ & 4898.09 & 4713.85 & $918.90 \mathrm{~N}$ & $32.13 \mathrm{E}$ & 58.5 & 1029.37 \\
\hline
\end{tabular}




\begin{tabular}{|c|c|c|c|c|c|c|c|c|c|c|}
\hline 7208.01 & 6502.9 & $758.72 \mathrm{~N}$ & $1281.44 \mathrm{~W}$ & $\begin{array}{l}\text { W \#1 - well Ab- } \\
\text { dulrahmanOFF3 }\end{array}$ & 6675.42 & 6267.98 & $1564.40 \mathrm{~N}$ & $455.71 \mathrm{~W}$ & 82 & 1177.35 \\
\hline 7381.89 & 6545.82 & $853.43 \mathrm{~N}$ & $1420.75 \mathrm{~W}$ & $\begin{array}{l}\text { W \#1 - well Ab- } \\
\text { dulrahmanOFF3 }\end{array}$ & 6800.09 & 6345.1 & $1645.43 \mathrm{~N}$ & $510.69 \mathrm{~W}$ & 83.5 & 1223.02 \\
\hline 7665.62 & 6586.53 & $1011.18 \mathrm{~N}$ & $1652.79 \mathrm{~W}$ & $\begin{array}{l}\text { W \#1 - well Ab- } \\
\text { dulrahmanOFF3 }\end{array}$ & 7001.68 & 6453.83 & $1787.05 \mathrm{~N}$ & $604.04 \mathrm{~W}$ & 85.4 & 1311.28 \\
\hline 7988.68 & 6611.93 & $1192.26 \mathrm{~N}$ & $1919.13 W$ & $\begin{array}{l}\text { W \#1 - well Ab- } \\
\text { dulrahmanOFF3 }\end{array}$ & 7285.48 & 6572.95 & $2001.12 \mathrm{~N}$ & $746.72 \mathrm{~W}$ & 90.1 & 1424.9 \\
\hline 8087.66 & 6617.48 & $1247.58 \mathrm{~N}$ & $2001.02 \mathrm{~W}$ & $\begin{array}{l}\text { W \#1 - well Ab- } \\
\text { dulrahmanOFF3 }\end{array}$ & 7370.85 & 6599.28 & $2068.41 \mathrm{~N}$ & $792.13 \mathrm{~W}$ & 90 & 1461.33 \\
\hline 9071.29 & 6650.26 & $1795.60 \mathrm{~N}$ & $2817.18 \mathrm{~W}$ & $\begin{array}{l}\text { W \#1 - well Ab- } \\
\text { dulrahmanOFF3 }\end{array}$ & 8241.47 & 6663.9 & $2775.83 \mathrm{~N}$ & $1290.98 \mathrm{~W}$ & 91.3 & 1813.92 \\
\hline
\end{tabular}

In addition, Table 3 shows the clearance calculations between the subject well and the offset well 3 with respect of MD, TVD, North and East coordinates of both wells. In addition to the closest distance between the two wells. Accordingly, it shows that distance between the subject and offset well 3 were $2.63 \mathrm{ft}$ at $278.87 \mathrm{ft} \mathrm{MD}$ and increase gradually till reaching the target at $1813 \mathrm{ft}$ at $9071.29 \mathrm{MD}$. This separation is lower than in offset well 1 and offset well 2 which yield that well 3 had a high potential of the collision but it keeps away from the subject well by considering the OSF greater than 5 .

\subsection{Clearance between the Subject well and offset wells}

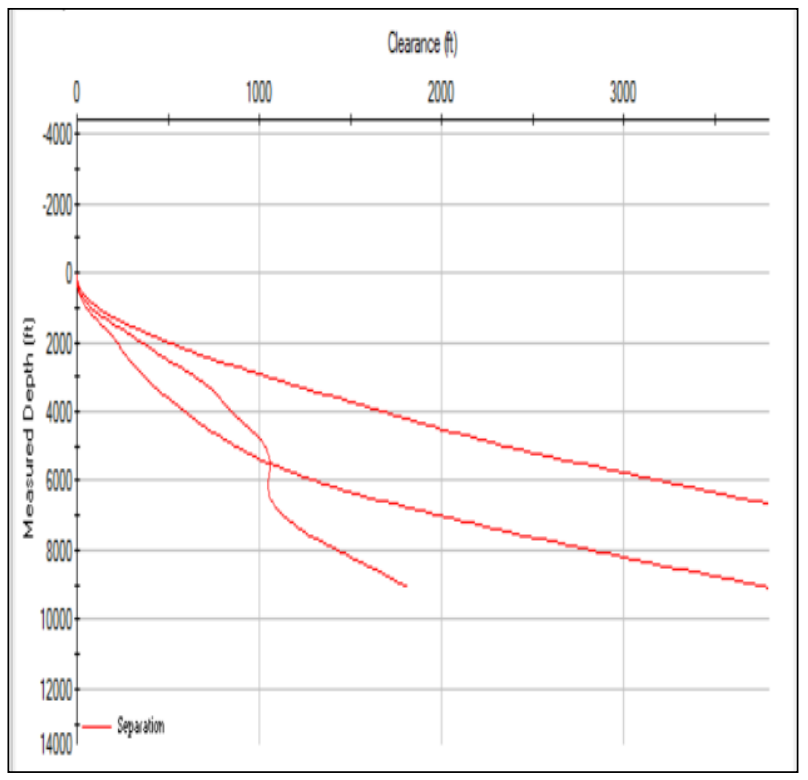

Fig. 3: Clearance vs. Measured Depth.

Fig. 3 shows the clearance results between the subject well and offset well 1, offset well 2 and offset well 3. It shows from the graph that MD of these offset wells and their distance from the subject or planned wells. This graph summarizes the results obtained in table 1 to table 3 as shows that the three wells keep away from the planned well with adequate separation that maintains the separation control rule.

\subsection{Anti-collision separation results}

Table 4: Anti-collision Proximity Calculations Summary

\begin{tabular}{|c|c|c|c|c|c|c|}
\hline \multicolumn{7}{|c|}{ Separation $\mathrm{Ct}-\mathrm{Ct}(\mathrm{ft})$} \\
\hline \multirow{8}{*}{ offset well 1} & & & & & $\mathrm{MD} \mathrm{ft}$ & TVD ft \\
\hline & 30 & 26.25 & 11 & 3.75 & 0 & 0 \\
\hline & 30 & 26.25 & 11 & 3.75 & 100 & 100 \\
\hline & 29.96 & 26.14 & 598 & 3.82 & 200 & 200 \\
\hline & 30.25 & 25.09 & $29 . .57$ & 5.16 & 400 & 400 \\
\hline & 46.73 & 39.1 & 17.08 & 7.63 & 700 & 699.63 \\
\hline & 75.39 & 64.8 & 15.99 & 10.59 & 1300 & 1292.6 \\
\hline & 9500 & 9109.14 & 377.8 & 390.86 & 11082.91 & 2500 \\
\hline \multirow{7}{*}{ offset well 2} & 10 & 6.25 & 3 & 3.75 & 0 & 0 \\
\hline & 10 & 6.25 & 3 & 3.75 & 0 & 0 \\
\hline & 7.81 & 1.78 & 3.5 & 6.03 & 500 & 499.98 \\
\hline & 7.01 & 0.66 & 2.6 & 6.35 & 600 & 599.88 \\
\hline & 11 & 3.99 & 3.91 & 7.01 & 700 & 699.63 \\
\hline & 40.75 & 30.51 & 8.84 & 10.24 & 1200 & 1196.07 \\
\hline & 9500 & 9063.35 & 290.61 & 436.65 & 11082.91 & 2500 \\
\hline \multirow{7}{*}{ Offset well3 } & 18.03 & 14.28 & 6.21 & 3.75 & 0 & 0 \\
\hline & 18.03 & 14.28 & 6.21 & 3.75 & 100 & 100 \\
\hline & 16.14 & 10.54 & 11.04 & 5.6 & 400 & 400 \\
\hline & 24.57 & 17.36 & 9.56 & 7.21 & 700 & 699.63 \\
\hline & 47.75 & 39.06 & 13.73 & 8.69 & 1200 & 1196.07 \\
\hline & 49.62 & 39.8 & 11.64 & 9.82 & 1300 & 1292.6 \\
\hline & 9500 & 9012.26 & 215.99 & 487.74 & 11082.91 & 2500 \\
\hline
\end{tabular}


Table 4 shows the anti-collision summary of the proximity calculation of the subject well and three offset wells. The proximity calculations were Separation centre to centre distance, allowable deviation separation factor and minimum allowable separation. These calculations are carried between the subject well and three offset wells 1,2 and 3 in terms of Measured depth (MD) and True Vertical Depth (TVD). The separation factor for offset well (1) ranges from 11 to 377.9 at the target depth. While the separation factor for offset well (2) ranges from 3 to 290.61 at the target depth. In addition to these wells, offset well (3) have a separation factor range from 6.21 to 215.99 at the target depth. From these data, the most risk well is offset well (2) as it is the closest well to the subject well. Furthermore, this is confirmed through minimum allowable separation as it ranges from $3.75-390.6 \mathrm{ft}, 3.75-436.65,3.75-487.74 \mathrm{ft}$ for offset wells 1,2 and 3 respectively.

Table 5: Anti-collision Proximity Calculations Summary

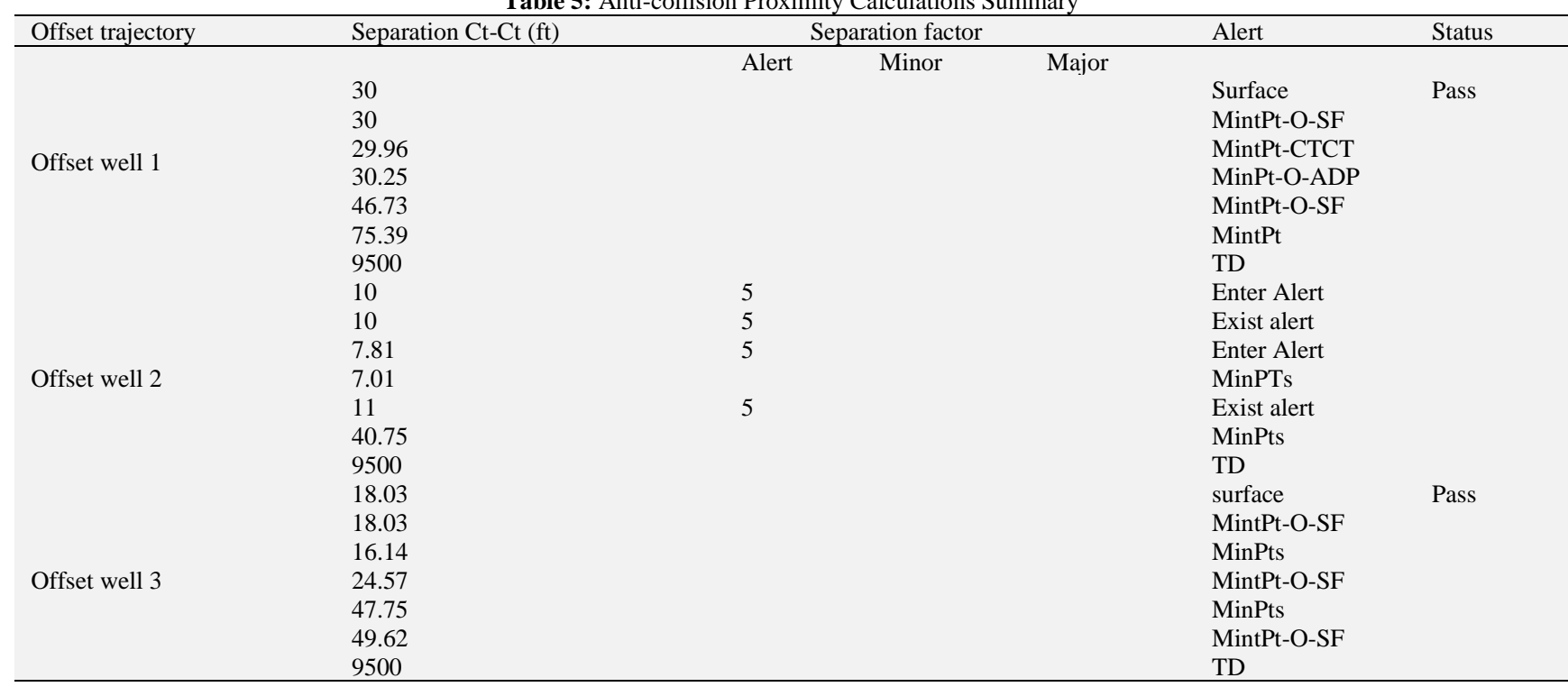

Table 5 shows the detailed anti-collision report for the subject well and the three offset wells. This report shows the centre to centre distance, separation factor (OSF), classification of the OSF according to the alerts and the status. Form the results, the OSF for offset well 1 and offset well is greater than 5 that drilled safely with an appropriate separation. While for offset well 2, the separation factor (OSF) is 5 along with MD depth from $500 \mathrm{ft}$ to $7000 \mathrm{ft}$. In addition, according to the rules for OSF, this well enters the alert zone at 500 and exit at $7000 \mathrm{ft}$. Moreover, the subject well is saved from the collision with offset well 2 through consideration of the novel OSF method.

\subsection{Graphical result of anti-collision planning}

\subsubsection{Travelling cylinder plots}

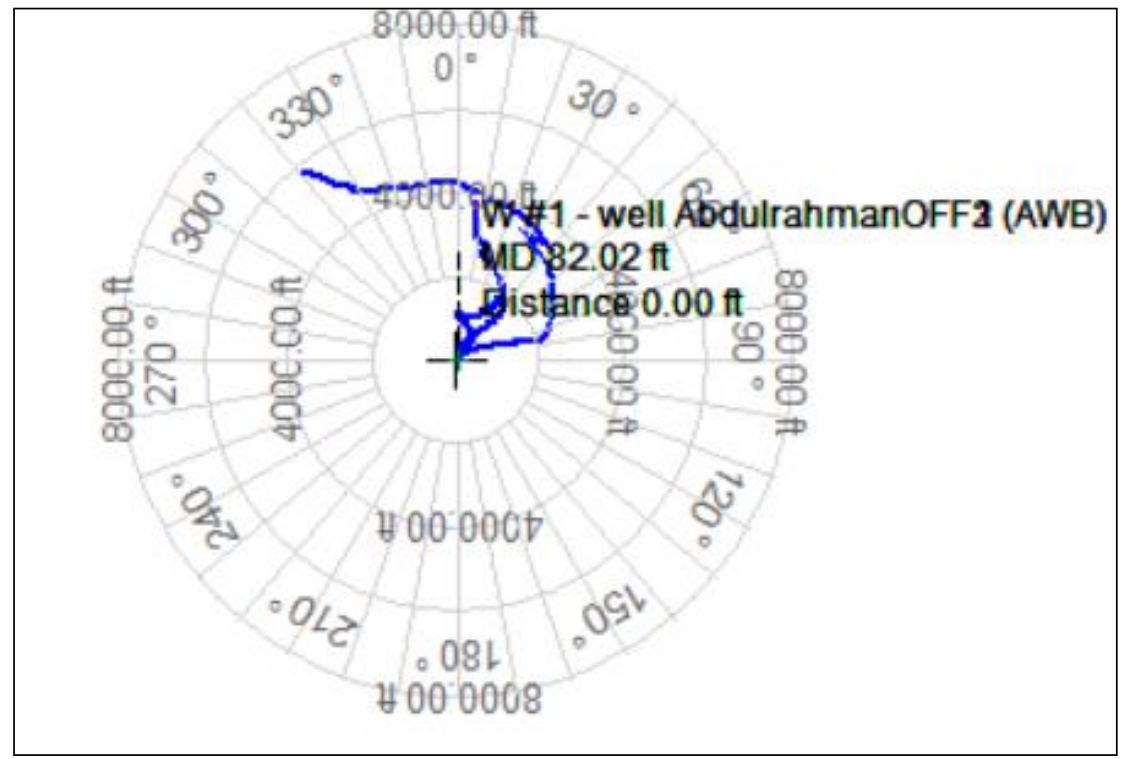

Fig. 4: Travelling Cylinder Plot of Anti-Collision Well Planning.

Fig.4 Shows the travelling cylinder plot for the subject well and the three offset wells. The plot shows the MD of these wells and their azimuth in comparison with the subject well. This plot yield that subjects well is drilled safely with appropriate separation from the offset wells from 120 degrees to 320-degree Azimuth at the target depth. 


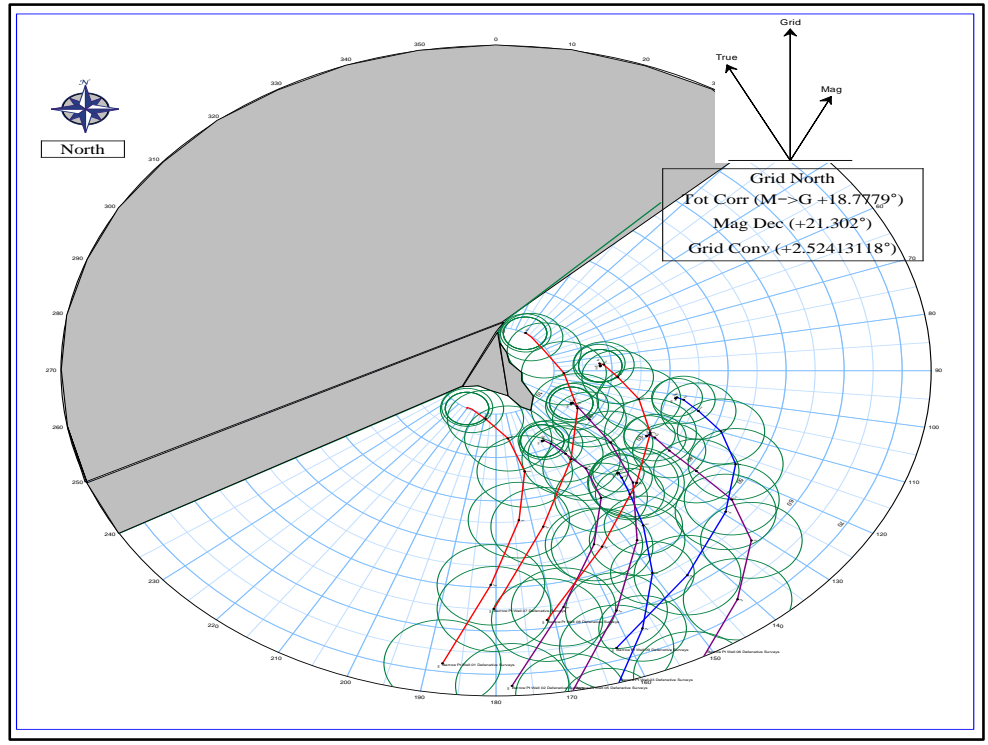

Fig. 5: Traveling Cylinder Plot with Drilling Tunnel.

Moreover, Fig.5 shows the travelling cylinder plot of these offset wells with respect to subject well as shown in Fig.4 but have drilling tunnel that comparing the position of the projection versus the NO-GO zones. The circles of NO-GO are plotted around the trajectories of the offset wells and subject well for a depth through using the circle radius equal to minimum allowable separation MAS as calculated in proximity calculations.

\subsubsection{Spider plot}

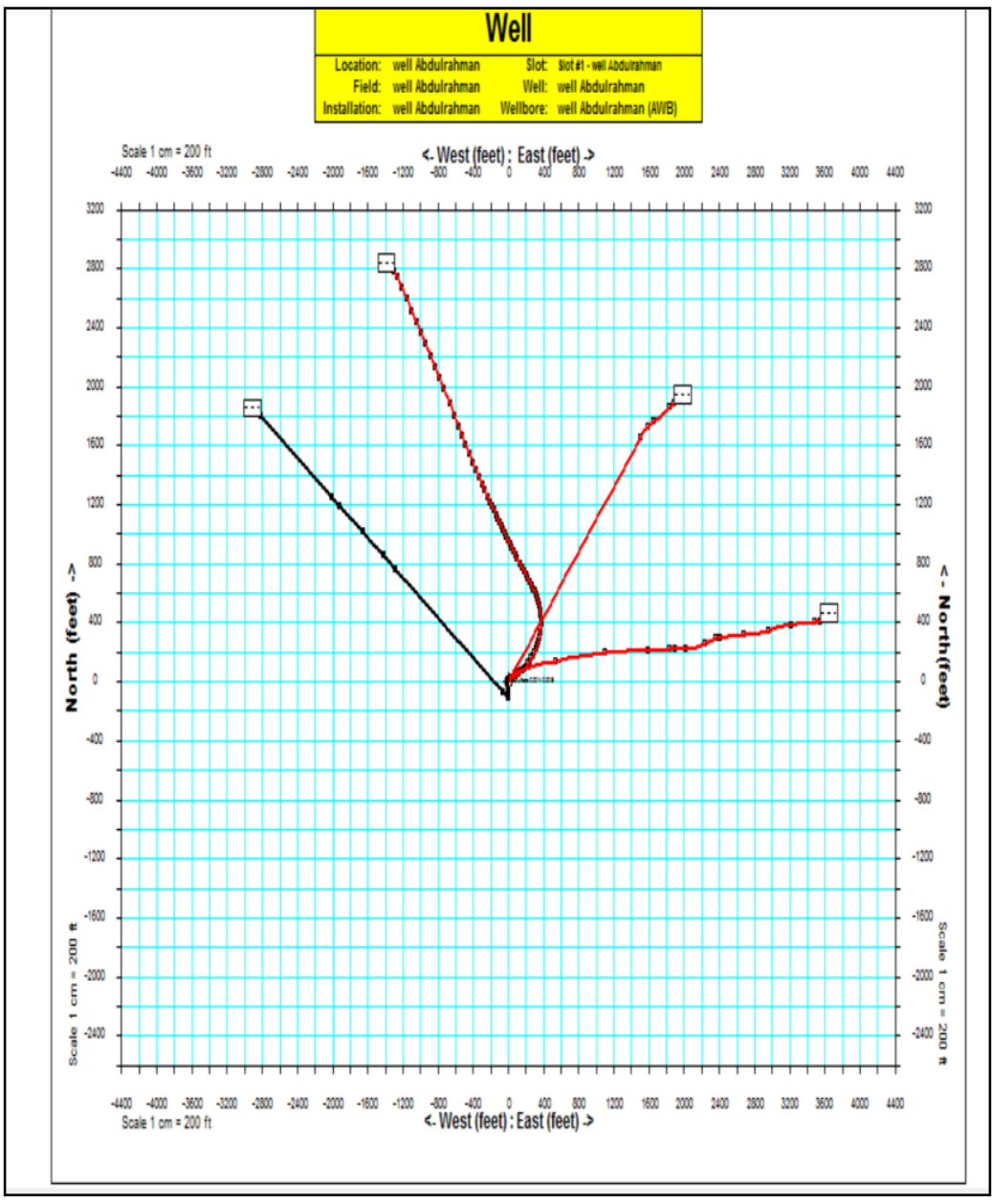

Fig. 6: Spider plot.

Fig.6 shows spider plot result that shows the offset wells in red line and the subject well in black colour that shows the direction of the offset wells and planned wells with respect to the north and west or east coordinates. From this plot, the subject well has clear distance from the offset wells at 0 degrees at the surface to 3200-degree West at target and 0 at 0 degrees at the surface to 1800-degree North at target. 


\subsection{Optimization of the drilling performance results}

\section{Total Performance}

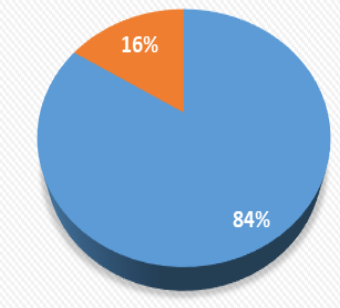

- Total Rotary VS Sliding

Fig. 7: Total Performance of Drilling.

Fig.7 shows the comparison between the total rotation versus the sliding of the drill string due to the adequate planning of the anti-collision well, as shown the rotation represents $84 \%$ while the sliding is $16 \%$. This result indicates good drilling performance as the rotation is greater than sliding due to the sliding cause more friction force on the drill string, cause limitation of the weight on the bit which results in lower ROP. While the Rotation results in better hole cleaning, lower friction, higher ROP and higher WOB. Accordingly, the drilling time is faster with greater Rotation than sliding.

\subsection{Economic results}

\subsubsection{Progress and cost chart}

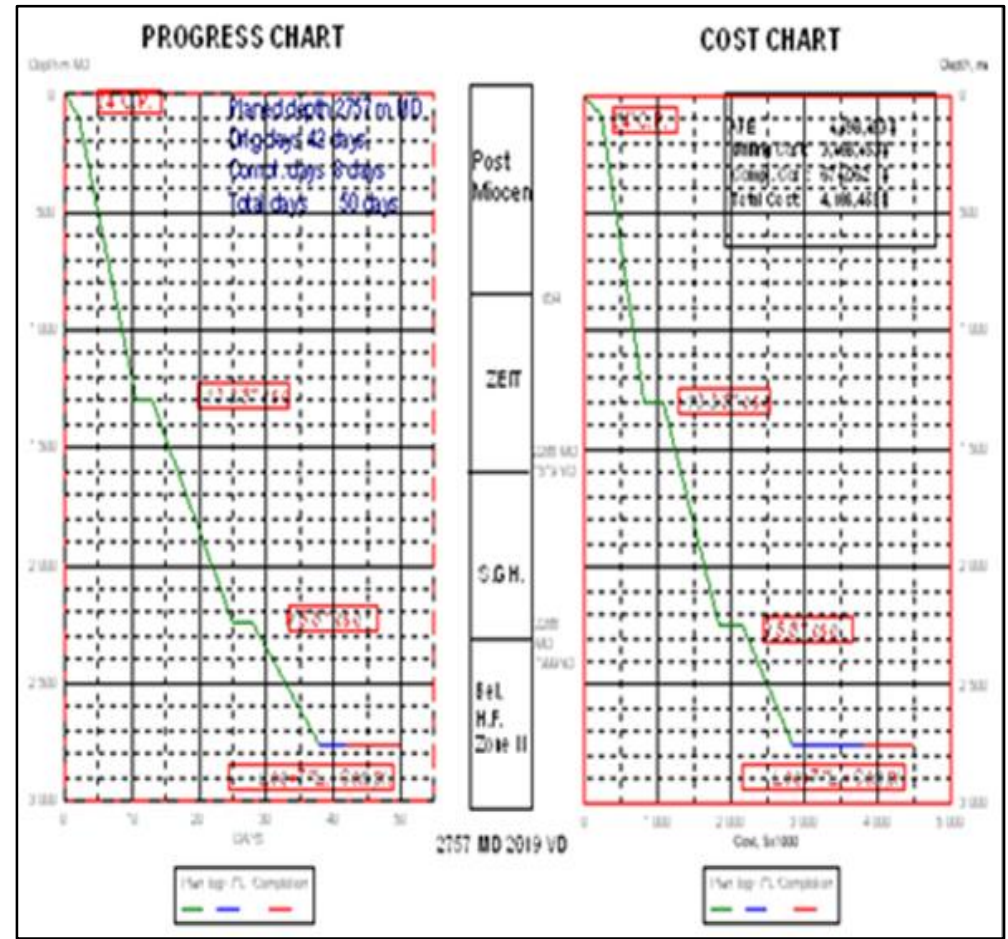

Fig. 8: Progress and Cost Chart.

Fig. 8 shows the progress and cost chart. The progress chart shows in the $\mathrm{x}$-axis days and the $\mathrm{Y}$-axis depth which shows the progress of drilling operation about 50 days with the total cost of 4,498,453 K \$.

\subsubsection{Economic analysis}

Table 6: Economics Results

\begin{tabular}{llllll}
\hline NPV \$ & IRR & ROI & DROI & PAY-OUT TIME & THE LIFETIME OF THE PROJECT \\
\hline $305,712,206.06 \$$ & $105 \%$ & 8.55 & 4.57 & 0.7 years & 34 Years \\
\hline
\end{tabular}

The table 6 shows the NPV of 305.7 Million \$ and the lifetime of the project will be 34 years with the internal rate of return of $105 \%$, rate of return of 8.55 and the discounted rate of return of 4.57 . 


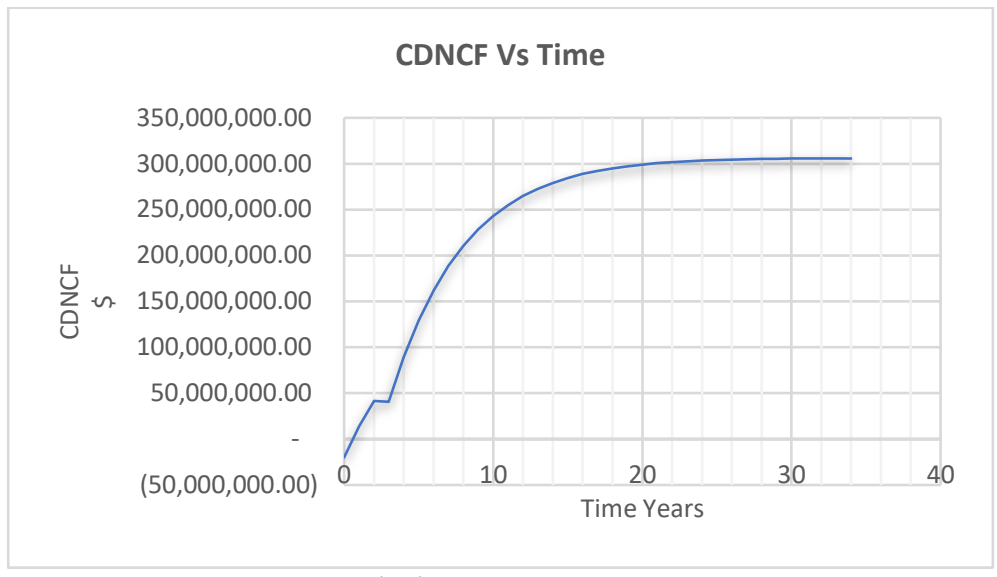

Fig. 9: CDNCF vs Time.

It can be shown form the Fig. 9 pay-out time is obtained at 0.7 years which is the point of intersection where the NPV becomes zero.

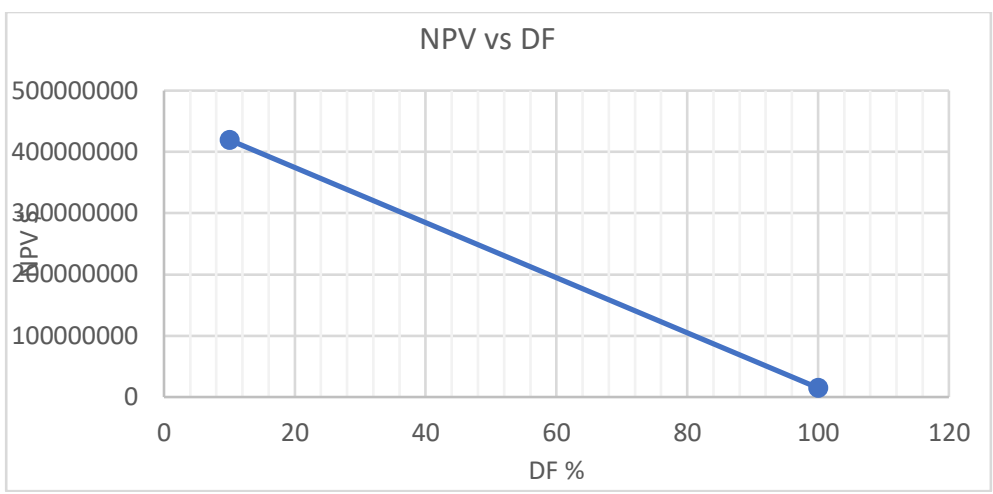

Fig. 10: NPV vs DF.

Fig.10 shows the discount factor percentage in the X-axis the net present value (NPV) in the Y-axis. The intersection of the line with the Discount factor percentage that results in the NPV to be zero. This IRR gives an indication of the profitability of the project where appear in this plot at $105 \%$.

\section{Conclusion}

The proposed well is drilled in the neighbouring of Offset well 1, Offset well 2 and Offset well 3 safely according to the separation rules. The anti-collision summary shows that the well is entered only the alert zone and redesign the plan to exit from the alert zone to drill safely and avoid the collision of the offset wells. The travelling cylinder plot that shows the clearance of the planned well and the offset wells through the measured depth and the azimuth of the wells which helps in avoid the collision with offset wells. Similarly, the spider plot shows the projection of the horizontal plane that appear the wells that exist in a given area as if not isolation on it as the earth was transparent which is used to avoid the collision between the wells an ensure the separation between the wells is maintained similarly to the travelling cylinder. The drilling is optimized as the rotation represent $84 \%$ while the sliding is $16 \%$. This result indicates good drilling performance as the rotation is greater than sliding due to the sliding cause more friction force on the drill string. The Drilling and completion time of the well is 50 days and along with the cumulative cost of 4,498,453 K \$. Finally, economic analysis shows positive income as NPV of 305.7 Million $\$$ and the lifetime of the project will be 34 years with the internal rate of return of $105 \%$, rate of return of 8.55 and the discounted rate of return of 4.57 .

\section{References}

[1] Bernt Aadnoy, Iain Cooper, Stefan Miska, Robert F. Mitchell, and Michael L. Payne. (2009). Advanced Drilling and Well Technology. United States: Society of Petroleum Engineers.

[2] Adam T. Bourgoyne, J. K. (1986). Applied Drilling Engineering. Society of Petroleum Engineers.

[3] Poedjono, B. (2010). Case Studies in the Application of an Effective Anti-collision Risk Management Standard. SPE, 19. https://doi.org/10.2118/121040-MS.

[4] Chen, D. C.-K. (2007). Integrated BHA Modeling Delivers Optimal BHA Design. SPE/IADC Middle East Drilling and Technology Conference (p. 11). Cairo: SPE. https://doi.org/10.2118/106935-MS

[5] Spidle, K. (2012). Drilling Office X (DOX) Technical Manual / Anti-Collision. Schlumberger, 41.

[6] Adly, E. (2007). Rotary Steerable System Technology Case Studies in the Canadian Foothills. American Association of Drilling Engineers, 9.

[7] Montecinos, j. (2012). DIRECTIONAL CONTROL \& SURVEYING PROCEDURES. ENI, 67.

[8] Wilson, G. (1968). Radius of Curvature Method for Computing Directional Surveys. SPWLA 9th Annual Logging Symposium (p. 11). Louisiana: SPE. 